\title{
Psychometric Testing of the Japanese Version of the Daily Record of Severity of Problems Among Japanese Women
}

This article was published in the following Dove Press journal: International Journal of Women's Health

\author{
Takashi Takeda $\mathbb{D}^{\prime}$ \\ Sayaka Kai ${ }^{1,2}$ \\ Kana Yoshimi ${ }^{1}$ \\ 'Division of Women's Health, Research \\ Institute of Traditional Asian Medicine, \\ Kindai University, Osaka-Sayama, Osaka, \\ 589-85I I, Japan; ${ }^{2}$ Department of \\ Obstetrics and Gynecology, Kindai \\ University Faculty of Medicine, \\ Osaka-Sayama, Osaka, 589-85I I, Japan
}

Correspondence: Takashi Takeda Division of Women's Health, Research Institute of Traditional Asian Medicine, Kindai University, 377-2 Ohno-Higashi, Osaka-Sayama, Osaka, 589-85II, Japan

Tel +8I-72-366-022I (Ext. 3393)

$\mathrm{Fax}+81-72-366-666 \mid$

Email take@med.kindai.ac.jp
Purpose: The Daily Record of Severity of Problems (DRSP) is commonly used to assess premenstrual syndrome and premenstrual dysphoric disorder throughout the world. The aim of this study was to assess the validity and reliability of the Japanese version of the DRSP (DRSP-J).

Materials and Methods: We analyzed 324 women with regular menstrual cycles who completed the DRSP-J and the Premenstrual Symptoms Questionnaire (PSQ). They had all applied to participate in an ongoing study for the treatment of their premenstrual symptoms. The DRSP-J was examined for evidence of reliability and validity. To examine reliability, we assessed Cronbach's $\alpha$, a measure of internal consistency, and test-retest reliability. We assessed structural validity with principal component factor analysis (PCA) and confirmatory factor analysis (CFA). We used PSQ total score to examine concurrent validity.

Results: Cronbach's $\alpha$ for DRSP total score was 0.97. DRSP total score and individual items showed high test-retest reliability. PCA showed a two-factor model describing mood and behavioral and physical symptoms. CFA showed that the two-factor model derived from the PCA was an acceptably good fit. DRSP total score correlated moderately with PSQ total score $(\mathrm{r}=0.42)$.

Conclusion: The DRSP-J showed reliable and valid measures of premenstrual symptoms in Japanese women.

Keywords: premenstrual syndrome, premenstrual disorders, psychometric testing, validity

\section{Introduction}

Premenstrual symptoms affect many women with a wide range of mood, behavioral, and physical symptoms that are limited to the luteal phase. ${ }^{1}$ Premenstrual disorders (PMDs) include premenstrual syndrome (PMS) and premenstrual dysphoric disorder (PMDD). ${ }^{2}$ Epidemiologic surveys have shown the high prevalence of premenstrual symptoms among menstruating women (80-90\%). ${ }^{3}$ A severe form of PMS is diagnosed as PMDD mainly by its psychiatric symptoms, according to the Diagnostic and Statistical Manual of Mental Disorders (DSM). ${ }^{4}$

The Daily Record of Severity of Problems (DRSP) is a prospective daily charting which is widely used for the diagnosis and evaluation of PMDs. The DRSP was developed to aid the assessment of PMDD according to the DSM criteria. Drospirenone-containing oral contraceptives and serotonin reuptake inhibitors are recognized as the standard therapy for PMDs in the world. ${ }^{5-7}$ The US food and drug administration approved these drugs for PMDD. In the clinical trials 
to show the efficacy of these drugs, DRSP scores were used as the primary endpoint. ${ }^{8-11}$ The DRSP was originally developed in English by Endicott ${ }^{12}$ and has since been translated into many languages, including German, ${ }^{13}$ Portuguese, ${ }^{14}$ and Chinese. ${ }^{15}$ We translated the DRSP into Japanese for the purpose of this clinical study, ${ }^{16}$ which is the first to use the Japanese version of the DRSP (DRSP-J). ${ }^{17}$ The DRSP-J was linguistically validated according to the general cross-cultural adaptation process, but its reliability and validity have not been systematically evaluated.

The aims of this study were to assess the validity and reliability of a patient-reported outcome measurement tool for premenstrual symptoms, the DRSP-J.

\section{Materials and Methods}

\section{Settings and Participants}

We analyzed 324 Japanese women with regular menstrual cycles who completed the DRSP-J and the Premenstrual Symptoms Questionnaire (PSQ). They applied to participate in an ongoing clinical trial for treatment of their premenstrual symptoms with a Royal jelly capsule supplement. Participants were recruited by advertisement in local free papers and by posters displayed in Kindai University Hospital. This trial was registered with the University Hospital Medical Information Network (UMIN) center (ID UMIN000040055). Data from the first screening cycle were used for this study.

To be eligible for the trial, women needed to fulfil all of the following criteria: age 20 to 45 years; presence of premenstrual symptoms with a suspected case of 'moderate-tosevere PMS' or "PMDD" according to the PSQ; ${ }^{18}$ regular menstrual cycles (25-38 days); no oral conceptive pill use for 4 weeks before study entry; no concurrent therapy for PMS, including antidepressants, herbs, and supplements, for 4 weeks before study entry; and written informed consent. Study subjects were excluded for the following reasons: presence of neuropsychiatric disorders; presence of very severe interference with work, usual activities, or relationships by premenstrual symptoms; serious hepatic disease, heart disease, kidney disease, or malignant disease.

\section{Questionnaire}

\section{Japanese Version of the Daily Record of Severity of Problems}

Japanese translation of the DRSP and the use of this Japanese version of the DRSP for our clinical trial were permitted by Endicott, who originally developed the English version. ${ }^{16}$ We translated the DRSP into Japanese, and it was linguistically validated according to the general cross-cultural adaptation process in our previous study. ${ }^{17}$ The back-translated version was verified for accuracy and equivalence by Endicott. The DRSP comprises 21 items regarding emotional and physical premenstrual symptoms and three items regarding functional impairment of social and life activities, all of which are rated for their severity on a 6-point scale (1, not at all; 6, extreme). All the symptoms are listed in Table 1.

The participants started the daily rating from the first day of their menstruation (day 1). The DRSP total score was calculated with the sum of the 21 premenstrual symptoms and ranges from 21 to 126 . The DRSP negative mood score was calculated as the sum of the eight core mood premenstrual symptoms (depressed/sad/blue, hopeless, worthless/guilty, anxious/tense/on edge, mood swings, sensitive to rejection, anger/irritability, conflict/ problems) and ranges from 8 to $48 .{ }^{19}$ These symptoms correspond to the core mood symptoms in the DSM-5 criteria for PMDD. Luteal phase scores were calculated by averaging the ratings of cycle day -5 to -1 . Follicular phase scores were calculated by averaging the ratings of cycle day 6 to 10 . We selected as participants those who answered the questionnaire at least 4 days in both the follicular and luteal phases.

\section{The Premenstrual Symptoms Questionnaire}

The PSQ was developed in our previous study to screen for premenstrual symptoms. ${ }^{18}$ In this patient-reported outcome measurement (PROM), the PMDD criteria from the DSM are translated into a rating scale with degrees of severity described in Japanese. The PSQ is useful and its reliability and validity have been fully evaluated in our previous studies. ${ }^{18,20,21}$ Therefore, we selected the PSQ to study the concurrent validity of the DRSP-J.

The PSQ asks,

Within the last 3 months, have you experienced the following premenstrual symptoms starting during the week before menses and stopping a few days after the onset of menses?

The premenstrual symptoms listed are

(1) depressed mood, (2) anxiety or tension, (3) tearfulness,

(4) anger or irritability, (5) decreased interest in work, home, or social activities, (6) difficulty concentrating, (7) fatigue or lack of energy, (8) overeating or food cravings, 
Table I Test-Retest Intraclass Correlation Coefficients of DRSP Total Scores and Items $(\mathrm{N}=324)^{*}$

\begin{tabular}{|c|c|c|c|}
\hline & $\begin{array}{l}\text { Mid- } \\
\text { Follicular } \\
\text { Phase Day } 9 \\
\text { vs Day I0 }\end{array}$ & $\begin{array}{l}\text { Late Luteal } \\
\text { Phase Day } \\
\text {-2 vs Day - I }\end{array}$ & $\begin{array}{l}\text { Change } \\
\text { Late } \\
\text { Luteal-Mid } \\
\text { Follicular }\end{array}$ \\
\hline Total score & 0.87 & 0.89 & 0.84 \\
\hline $\begin{array}{l}\text { Negative mood } \\
\text { score }\end{array}$ & 0.81 & 0.85 & 0.81 \\
\hline la & 0.69 & 0.76 & 0.65 \\
\hline $\mathrm{Ib}$ & 0.68 & 0.84 & 0.77 \\
\hline Ic & 0.71 & 0.78 & 0.70 \\
\hline 2 & 0.79 & 0.73 & 0.66 \\
\hline $3 a$ & 0.77 & 0.77 & 0.74 \\
\hline $3 b$ & 0.75 & 0.80 & 0.74 \\
\hline $4 a$ & 0.79 & 0.74 & 0.70 \\
\hline $4 b$ & 0.68 & 0.76 & 0.67 \\
\hline 5 & 0.72 & 0.78 & 0.68 \\
\hline 6 & 0.80 & 0.74 & 0.67 \\
\hline 7 & 0.71 & 0.72 & 0.58 \\
\hline $8 a$ & 0.73 & 0.79 & 0.70 \\
\hline $8 b$ & 0.74 & 0.82 & 0.73 \\
\hline $9 a$ & 0.70 & 0.77 & 0.65 \\
\hline $9 b$ & 0.76 & 0.75 & 0.63 \\
\hline $10 \mathrm{a}$ & 0.69 & 0.83 & 0.74 \\
\hline $10 \mathrm{~b}$ & 0.58 & 0.73 & 0.63 \\
\hline Ila & 0.88 & 0.84 & 0.82 \\
\hline $\mathrm{Ilb}$ & 0.85 & 0.81 & 0.79 \\
\hline IIc & 0.52 & 0.70 & 0.56 \\
\hline IId & 0.75 & 0.84 & 0.70 \\
\hline
\end{tabular}

Notes: Ia, depressed/sad/blue; Ib, hopeless; Ic, worthless/guilty; 2, anxious/tense/on edge; 3a, mood swings; 3b, sensitive to rejection; $4 \mathrm{a}$, anger/irritability; 4b, conflict/ problems; 5 , less interest; 6 , difficulty concentrating; 7, lethargic/tired/fatigued; 8a, increased appetite; 8b, crave specific foods; 9a, sleep more; 9b, trouble sleeping; 10a, overwhelmed, cannot cope; IOb, out of control; I la, breast tenderness; I l b, breast swelling/“bloated;" I Ic, headache; I Id, joint/muscle pain. *All $P<0.00$ I.

(9) insomnia or hypersomnia, (10) feeling overwhelmed, and (11) physical symptoms such as tender breasts, feeling of bloating, headache, joint or muscle pain, or weight gain.

These 11 symptoms are listed in the DSM criteria for PMDD. The PSQ also asks whether the premenstrual symptoms experienced interfere with (a) work efficiency or productivity, or home responsibilities; (b) social activities; or (c) relationships with coworkers or family. These three items measuring functional impairment of social and life activities were same as items in the DRSP-J. ${ }^{12,17}$ In this study, participants were asked to rate the severity of premenstrual symptoms on the first day of the DRSP recording and these symptoms' interference with activities as 1 (not at all), 2 (mild), 3 (moderate), or 4 (severe). The PSQ total score was calculated as the sum of 14 items and ranges from 14 to 56 .

\section{Statistical Analysis}

Means and standard deviations (SD) were calculated for the age of participants.

The internal consistency reliabilities of the DRSP-J were assessed with Cronbach's $\alpha$ coefficient. The day-to-day testretest reliability was assessed with the intraclass correlation coefficients (ICC). Adjacent days within the different phase (mid-follicular phase day 9 vs day 10 and late luteal phase day -2 vs day -1 ) were selected. The changes, calculated as late luteal minus mid follicular (day -2 minus day 9 vs day -1 minus day 10), were also assessed.

The factor structure of the DRSP-J was examined in a principal component factor analysis (PCA) with Promax rotation using the luteal phase scores. The eigenvalue $>1.0$ criteria and scree plot criteria were used to determine the number of factors to extract.

To confirm the factorial validity, we performed a confirmatory factor analysis (CFA) with two models. The first model was based on DSM-5 criteria for PMDD. In this model, two dimensions were assumed. The first dimension was the core mood dimension, which was essential for the diagnosis of PMDD in DSM-5 criteria. The second dimension was the other symptoms, which was mainly behavioral and physical dimension. The second model was based on the PCA model. In the CFA, model fit was evaluated using fit indices: the chisquare/degree of freedom $\left(\chi^{2} / \mathrm{df}\right)$, the goodness-of-fit index (GFI), the Tucker-Lewis Index (TLI), the comparative fit index (CFI), the root mean square residual error of approximation (RMSEA), the standardized root mean square residual (SRMR), and the Akaike's information criterion (AIC). $\chi^{2} / \mathrm{df}$ values $<5$ indicate a good fit. ${ }^{22}$ GFI values $>0.90$ indicate a good fit. ${ }^{23}$ TLI and CFI values close to 0.95 indicate a relatively good fit. ${ }^{24}$ A RMSEA value $<0.08$ suggests a good fit, $0.08-0.10$ a moderate fit, and $>0.10$ a poor fit. SRMR values $<0.09$ suggest a good fit. ${ }^{23}$ 
Table 2 Internal Consistency (Cronbach's Alpha) for the Summary Scores of DRSP (N=324)

\begin{tabular}{|l|l|l|l|}
\hline & Luteal Phase Score & Follicular Phase Score & Luteal Phase Score Minus Follicular Phase Score \\
\hline Total score & 0.97 & 0.98 & 0.96 \\
\hline Negative mood score & 0.97 & 0.97 & 0.97 \\
\hline Factor I: Mood & 0.98 & 0.98 & 0.98 \\
\hline Factor 2: Behavior/Physical & 0.87 & 0.91 & 0.86 \\
\hline
\end{tabular}

Abbreviation: DRSP, Daily Record of Severity of Problems.

A smaller AIC indicated a better adjustment between goodness-of-fit and parsimony.

Correlations were analyzed using Spearman's rank correlation coefficient.

Statistical analyses except for the CFA and the ICC were performed using JMP Pro 15.2.0 (SAS, Cary, NC, USA). The CFA was performed using IBM SPSS Amos 26 (IBM Corp., New York, USA). ICC was calculated using the Excel add-in software BellCurve for Excel 3.2.0 (Social Survey Research Information Co., Ltd., Tokyo, Japan). Statistical significance was set at $P<0.05$ (two-tailed tests).

\section{Results}

The mean age of participants was 35.2 years (SD 6.3, range $20-45$ years).

The Cronbach's $\alpha$ for the DRSP total score was 0.97 in the luteal phase and 0.98 in the follicular phase, which indicated very good internal consistency (Table 2). In the clinical trials to assess the efficacy of drugs for PMDs, the evaluation of the score change from the mid follicular phase to the late luteal phase was needed. ${ }^{8-11}$ Therefore, we further calculated the DRSP total scores for the luteal phase minus follicular phase. The Cronbach's $\alpha$ for this score was 0.96 , which indicated very good internal consistency. The Cronbach's $\alpha$ for the DRSP negative mood score also demonstrated good internal consistency in the luteal phase, follicular phase, and the luteal phase minus follicular phase.

The test-retest reliability for the DRSP-J is shown in Table 1. The test-retest values of the DRSP total score and the DRSP negative mood score in the follicular phase, the luteal phase, and the change score were all above 0.80 . Most of the data were within the very good range, and all the luteal phase scores were all above 0.70 .

To explore the factor structure of DRSP-J, we performed a PCA. The eigenvalue $>1.0$ criteria showed two factors and the scree plot also identified two factors. Table 3 shows the factor coefficients DRSP-J after Promax rotation. The first factor (Factor 1) explained
$59.15 \%$ of data variance and the second factor (Factor 2) $37.44 \%$. Factor 1 can be interpreted as a mood dimension and Factor 2 as a behavior/physical dimension.

The Cronbach's $\alpha$ for each factor is shown in Table 2. All the data showed higher than 0.80 , which indicates good internal consistency.

To analyze the factorial validity of the DRSP-J, we performed a CFA with two models (Supplemental Material). Model 1, which was based on DSM-5 criteria for PMDD, showed a relatively good fit on several indicators. Model 2, which was derived from a PCA, showed a better fit than model 1 (Table 4). The superiority of model 2 was proved by the higher GFI, TLI, and CFI values and the lower $\chi^{2} / \mathrm{df}$, RMSEA, and AIC values. This model achieved acceptable values of the goodnessof-fit indices for the DRSP-J.

Next, we analyzed the consistency of the evaluation of premenstrual symptoms between the DRSP and the PSQ (Table 5). The DRSP total score and the DRSP negative mood score were found to be moderately correlated with the PSQ total score (Spearman's $r=0.42$ for total score and 0.39 for negative mood score, $P<0.0001)$. The correlation of Factor 1 (mood) and the PSQ total score was similar to that of the DRSP total score. The correlation of Factor 2 (behavior/physical) and the PSQ total score was less than that of DRSP total score but was within the moderate range.

\section{Discussion}

The present study evaluated the DRSP-J, a PROM for PMDs. We found strong evidence of validity and reliability for the questionnaire. We could find only two studies on PubMed that analyzed the reliability and validity of the DRSP. One uses the original English version ${ }^{12}$ and the other uses the Chinese version. ${ }^{15}$

The Cronbach's $\alpha$ of the DRSP-J total score for lateluteal minus mid-follicular change (0.96) showed a high degree of internal consistency. This value was as high as 
Table 3 Factor Coefficients of DRSP After Promax Rotation $(\mathrm{N}=324)$

\begin{tabular}{|c|c|c|}
\hline & $\begin{array}{l}\text { Factor I } \\
\text { (Mood) }\end{array}$ & $\begin{array}{c}\text { Factor } 2 \\
\text { (Behavior/Physical) }\end{array}$ \\
\hline Ic & 0.94 & -0.03 \\
\hline $3 b$ & 0.93 & 0.00 \\
\hline $3 a$ & 0.93 & 0.00 \\
\hline $\mathrm{la}$ & 0.92 & 0.00 \\
\hline $\mathrm{Ib}$ & 0.92 & -0.04 \\
\hline $4 b$ & 0.91 & -0.02 \\
\hline $10 \mathrm{a}$ & 0.90 & 0.02 \\
\hline 2 & 0.88 & 0.03 \\
\hline $10 \mathrm{~b}$ & 0.79 & 0.10 \\
\hline 5 & 0.78 & 0.07 \\
\hline $4 a$ & 0.78 & 0.17 \\
\hline 7 & 0.69 & 0.28 \\
\hline 6 & 0.68 & 0.28 \\
\hline $\mathrm{IIb}$ & -0.10 & 0.91 \\
\hline Ila & -0.17 & 0.84 \\
\hline $8 a$ & 0.19 & 0.64 \\
\hline $8 b$ & 0.26 & 0.54 \\
\hline $9 a$ & 0.36 & 0.52 \\
\hline IId & 0.18 & 0.51 \\
\hline $9 b$ & 0.38 & 0.44 \\
\hline IIC & 0.28 & 0.38 \\
\hline Eigenvalue & 13.01 & 1.66 \\
\hline $\begin{array}{l}\% \text { of variance } \\
\text { explained }\end{array}$ & 59.15 & 37.44 \\
\hline \multicolumn{3}{|l|}{$\begin{array}{l}\text { Correlation between } \\
\text { factors }\end{array}$} \\
\hline Factor I & 1.00 & \\
\hline Factor 2 & 0.57 & 1.00 \\
\hline
\end{tabular}

Notes: Value above 0.3 is expressed in bold. la, depressed/sad/blue; Ib, hopeless; Ic worthless/guilty; 2, anxious/tense/on edge; $3 \mathrm{a}$, mood swings; $3 \mathrm{~b}$, sensitive to rejection 4a, anger/irritability; 4b, conflict/problems; 5 , less interest; 6 , difficulty concentrating; 7 , lethargic/tired/fatigued; 8a, increased appetite; 8b, crave specific foods; 9a, sleep more; $9 \mathrm{~b}$, trouble sleeping; 10a, overwhelmed, cannot cope; I0b, out of control; I la, breast tenderness; I Ib, breast swelling/“bloated;” I lc, headache; I Id, joint/muscle pain.

that reported previously for the original DRSP $(0.95) .^{12}$ The test-retest reliability for DRSP-J was also high and as satisfactory as that of the original DRSP. The original study for the reliability and validity of the DRSP was tested in two types of study participants, those with few or no premenstrual problems and those with PMDD. ${ }^{12}$ The participants in the DRSP-J were those with premenstrual problems with varying degrees of severity not restricted to PMDD. The British Royal College of Obstetrics and Gynecology (RCOG) recommended the DRSP as the standard tool for tracking PMD symptoms not restricted to PMDD. ${ }^{6}$ Therefore, the severity of study participants' premenstrual symptoms as recorded in the DRSP-J was coincident with the RCOG recommendation.

One previous report on the results of a PCA of the Chinese version of the DRSP showed four factors: "mood," "behavior," "pain," and "physical symptoms.",15 In this model, physical symptoms were grouped into two factors, "pain" (headache, joint/muscle pain) and "physical symptoms" (breast tenderness, breast swelling/“bloated"). This discrepancy with our findings may be explained by differences in studies' recruitment methods. The participants in the Chinese version of the DRSP were all young, non-patient nursing students. However, the participants in the DRSP-J were all complaining of premenstrual symptoms and seeking treatment for their symptoms.

This is the first report about the CFA for PMDs using the DRSP. Our data indicated that a two-factor model derived from the PCA produced an acceptably good fit on indicators. Previous CFA reports about PMS also showed a two-factor model. ${ }^{25,26}$ Our CFA data further strengthen the construct validity of the DRSP-J.

One Japanese version of the DRSP has been reported independently by another group. ${ }^{27}$ They also reported the validity and reliability, but in their study the DRSP responses were not recorded daily but recorded only two days in the cycle (one day in the follicular phase and another day in the luteal phase). This study design was not in accordance with the usage of the original DRSP.

This study had several limitations. The main limitation was that the participants were limited to those with premenstrual problems. Therefore, it is impossible to evaluate the construct validity of the DRSP-J in those with no symptoms. But in the practical clinical setting, the DRSP would be used in the diagnosis of PMDs. This limitation is not critical because our main purpose in developing the DRSP-J is for its application in clinical treatment trials. A major strength of our study was the recruitment of subjects by advertisement in local free papers and by posters displayed in our university hospital. This allowed 
Table 4 Goodness-of-Fit Summary of the DRSP (Two-Factor Model) (N=324)

\begin{tabular}{|l|l|l|l|l|l|l|l|l|}
\hline Model & $\chi^{2} \mathbf{( d f )}$ & $\chi^{2}$ /df & GFI & TLI & CFI & RMSEA (90\% CI) & SRMR & AIC \\
\hline Model I & $748.59(175)$ & 4.28 & 0.819 & 0.911 & 0.926 & $0.101(0.093-0.108)$ & 0.046 & 860.59 \\
\hline Model 2 & $603.41(172)$ & 3.51 & 0.850 & 0.932 & 0.944 & $0.088(0.081-0.096)$ & 0.048 & 721.41 \\
\hline
\end{tabular}

Notes: Model I, two-factor (8 items + 13 items + 13 error covariance); Model 2, two-factor (13 items +8 items +16 error covariance).

Abbreviations: DRSP, Daily Record of Severity of Problems; $\chi^{2}$, chi-square; df, degrees of freedom; GFI, goodness-of-fit index; TLI, Tucker-Lewis index; CFI, comparative fit index; RMSEA, root mean square residual error of approximation; $\mathrm{Cl}$, confidence interval; SRMR, standardized root mean square residual; AIC, Akaike's information.

Table 5 Correlation Coefficients for the DRSP and the PSQ $(\mathrm{N}=324)$

\begin{tabular}{|l|l|}
\hline & PSQ Total Score Spearman's r \\
\hline Total score & $0.42^{*}$ \\
\hline Negative mood score & $0.39^{*}$ \\
\hline Factor I: Mood & $0.4 I^{*}$ \\
\hline Factor 2: Behavior/Physical & $0.35^{*}$ \\
\hline
\end{tabular}

Note: $* P<0.0001$.

Abbreviations: DRSP, Daily Record of Severity of Problems; PSQ, Premenstrual Symptoms Questionnaire.

the data to be applicable to any Japanese women with premenstrual problems.

In Japan, no medications for PMDs have been approved by the Ministry of Health, Labour and Welfare. We are attempting to demonstrate the efficacy of new drug labelling for the treatment of PMDs through a randomized, doubleblind, placebo-controlled trial in which we monitor PMD symptoms with the DRSP-J. The DRSP-J would contribute to the improvement of Japanese women's quality of life by assessing the efficacy of drugs in clinical trials.

\section{Conclusions}

This study presented precise evidence of the validity and acceptability of the DRSP-J as a measure of premenstrual symptoms in our study sample.

\section{Ethics Approval and Informed Consent}

The study was carried out in accordance with the principles outlined in the Declaration of Helsinki. The trial protocol was approved by the Ethics Committee of Kindai University (approval number: 28-234). Participants provided informed consent before entering the study.

\section{Acknowledgments}

We thank Lucy McClellan, MIA, from Edanz Group for editing a draft of this manuscript.

\section{Author Contributions}

All authors made substantial contributions to the conception and design, acquisition of data, or analysis and interpretation of the data; took part in drafting the article or revising it critically for important intellectual content; agreed to submit to the current journal; gave final approval of the version to be published; and agree to be accountable for all aspects of the work.

\section{Funding}

This work was supported in part by grants from YAMADA RESEARCH GRANT and Cyclic Innovation for Clinical Empowerment, AMED (19pc0101040h0001), Tokyo, Japan.

\section{Disclosure}

The authors have no conflicts of interest to declare.

\section{References}

1. Yonkers KA, O'Brien PM, Eriksson E. Premenstrual syndrome. Lancet. 2008;371(9619):1200-1210. doi:10.1016/S0140-6736(08) 60527-9

2. O'Brien PM, Backstrom T, Brown C, et al. Towards a consensus on diagnostic criteria, measurement and trial design of the premenstrual disorders: the ISPMD Montreal consensus. Arch Women Mental Health. 2011;14(1):13-21. doi:10.1007/s00737-010-0201-3

3. Angst J, Sellaro R, Merikangas KR, Endicott J. The epidemiology of perimenstrual psychological symptoms. Acta Psychiatr Scand. 2001;104(2):110-116. doi:10.1034/j.1600-0447.2001.00412.x

4. Association AP. Diagnostic and Statistical Manual of Mental Disorders: DSM-5. Arlington: American Psychiatric Association; 2013.

5. Hofmeister S, Bodden S. Premenstrual syndrome and premenstrual dysphoric disorder. Am Fam Physician. 2016;94(3):236-240.

6. Yonkers KA, Simoni MK. Premenstrual disorders. Am J Obstet Gynecol. 2018;218(1):68-74. doi:10.1016/j.ajog.2017.05.045

7. Kelderhouse K, Taylor JS. A review of treatment and management modalities for premenstrual dysphoric disorder. Nurs Women Health. 2013;17(4):294-305. doi:10.1111/1751-486X.12048

8. Pearlstein TB, Bachmann GA, Zacur HA, Yonkers KA. Treatment of premenstrual dysphoric disorder with a new drospirenone-containing oral contraceptive formulation. Contraception. 2005;72(6):414-421. doi:10.1016/j.contraception.2005.08.021

9. Pearlstein TB, Halbreich U, Batzar ED, et al. Psychosocial functioning in women with premenstrual dysphoric disorder before and after treatment with sertraline or placebo. J Clin Psychiatry. 2000;61 (2):101-109. doi:10.4088/JCP.v61n0205 
10. Yonkers KA, Halbreich U, Freeman E, et al. Symptomatic improvement of premenstrual dysphoric disorder with sertraline treatment. A randomized controlled trial. Sertraline Premenstrual Dysphoric Collaborative Study Group. JAMA. 1997;278(12):983-988. doi:10.1001/jama.1997.03550120043031

11. Shehata NA. Calcium versus oral contraceptive pills containing drospirenone for the treatment of mild to moderate premenstrual syndrome: a double blind randomized placebo controlled trial. Eur J Obstet Gynecol Reprod Biol. 2016;198:100-104. doi:10.1016/j. ejogrb.2016.01.015

12. Endicott J, Nee J, Harrison W. Daily Record of Severity of Problems (DRSP): reliability and validity. Arch Womens Ment Health. 2006;9 (1):41-49. doi:10.1007/s00737-005-0103-y

13. Schmidt K, Weber N, Steiner M, et al. A lecithin phosphatidylserine and phosphatidic acid complex (PAS) reduces symptoms of the premenstrual syndrome (PMS): results of a randomized, placebo-controlled, double-blind clinical trial. Clin Nutr ESPEN. 2018;24:22-30. doi:10.1016/j.clnesp.2018.01.067

14. Henz A, Ferreira CF, Oderich CL, et al. Premenstrual syndrome diagnosis: a comparative study between the Daily Record of Severity of Problems (DRSP) and the Premenstrual Symptoms Screening Tool (PSST). Rev Bras Ginecol Obstet. 2018;40 (1):20-25. doi:10.1055/s-0037-1608672

15. Wu L, He Z, Zhao $\mathrm{H}$, et al. Chinese version of daily record of severity of problems: reliability and validity. J Adv Nurs. 2013;69 (2):449-456. doi:10.1111/j.1365-2648.2012.06070.x

16. Takeda T, Shiina M, Chiba Y. Effectiveness of natural S-equol supplement for premenstrual symptoms: protocol of a randomised, double-blind, placebo-controlled trial. BMJ Open. 2018;8(7): e023314. doi:10.1136/bmjopen-2018-023314

17. Takeda T, Shiina M, Yamada K. Development of the Japanese version of the Daily Record of Severity of Problems (DRSP): translation and linguistic validation. Clin Gynecol Obstet. 2019;73(8):807-811.

18. Takeda T, Tasaka K, Sakata M, Murata Y. Prevalence of premenstrual syndrome and premenstrual dysphoric disorder in Japanese women. Arch Womens Ment Health. 2006;9(4):209-212. doi:10.1007/s00737-0060137-9
19. Bixo M, Ekberg K, Poromaa IS, et al. Treatment of premenstrual dysphoric disorder with the GABA(A) receptor modulating steroid antagonist Sepranolone (UC1010)-A randomized controlled trial. Psychoneuroendocrinology. 2017.

20. Takeda T, Koga S, Yaegashi N. Prevalence of premenstrual syndrome and premenstrual dysphoric disorder in Japanese high school students. Arch Women Mental Health. 2010;13(6):535-537. doi:10.1007/s00737-010-0181-3

21. Takeda T, Yoshimi K, Yamada K. Psychometric testing of the premenstrual symptoms questionnaire and the association between perceived injustice and premenstrual symptoms: a cross-sectional study among Japanese High School Students. Int J Womens Health. 2020;12:755-763. doi:10.2147/IJWH.S269392

22. Marsh HW, Hocevar D. Application of confirmatory factor analysis to the study of self-concept: first- and higher order factor models and their invariance across groups. Psychol Bull. 1985;97(3):562-582. doi:10.1037/0033-2909.97.3.562

23. Hooper DCJ, Mullen MR. Structural equation modelling: guidelines for determining model fit. Electron J Bus Res Methods. 2008;6(1):53-60.

24. Hu L-T, Bentler PM. Cutoff criteria for fit indexes in covariance structure analysis: conventional criteria versus new alternatives. Struct Equ Model. 1999;6(1):1-55. doi:10.1080/10705519909540118

25. Condon JT. Investigation of the reliability and factor structure of a questionnaire for assessment of the premenstrual syndrome. J Psychosomatic Res. 1993.

26. York R, Freeman EL, Lowery BA, Strauss JF 3rd. Characteristics of premenstrual syndrome. Obstet Gynecol. 1989.

27. Ikeda Y, Egawa M, Hiyoshi K, et al. Development of a Japanese version of the daily record of severity of problems for diagnosing premenstrual syndrome. Women Health Rep. 2020;1(1):11-16. doi:10.1089/whr.2019.0004

\section{Publish your work in this journal}

The International Journal of Women's Health is an international, peerreviewed open-access journal publishing original research, reports, editorials, reviews and commentaries on all aspects of women's healthcare including gynecology, obstetrics, and breast cancer. The manuscript management system is completely online and includes a very quick and fair peer-review system, which is all easy to use Visit http://www.dovepress.com/testimonials.php to read real quotes from published authors. 